INVESTIGACIÓN

https://doi.org/10.15198/seeci.2020.53.153-173

Recibido: 08/06/2020 --- Aceptado: 27/08/2020 --- Publicado: 15/11/2020

\title{
SECTOR TURÍSTICO: COMUNICACIÓN E INNOVACIÓN SOSTENIBLE
}

\section{TOURISM SECTOR: COMMUNICATION AND SUSTAINABLE INNOVATION}

(D) Almudena Barrientos-Báez: Escuela Universitaria de Turismo Iriarte (ULL). España.

almudenabarrientos@iriarteuniversidad.es

David Caldevilla-Domínguez: Universidad Complutense de Madrid. España. davidcaldevilla@ccinf.ucm.es

Andrea Cáceres Vizcaíno: Universidad de La Laguna. España.

caceresvizcainoandrea@gmail.com

Ema Gloria Sueia Val: Universidad de La Laguna. España.

gloriasueiaval@gmail.com

\section{RESUMEN:}

El presente artículo se desarrolla a partir de la premisa de analizar los medios y herramientas implementadas en España para la mejora y acondicionamiento del sector turístico respecto a la sostenibilidad. Se analizan las razones que han llevado al segmento de la hotelería a implementar medidas de cambio para la conservación del entorno y del patrimonio cultural español. Se pretende a su vez analizar las acciones llevadas a cabo en territorio europeo y nacional.

\section{PALABRAS CLAVE:}

Comunicación - España - Europa - Innovación sostenible - Sostenibilidad - Turismo.

\section{ABSTRACT:}

This research is developed based on the premise of analyzing the means and tools implemented in Spain for the improvement and conditioning of the tourism sector in 
relation to sustainability. The reasons that have led the hotel trade to implement measures of change for the conservation of the environment and the Spanish cultural heritage are analyzed. It is intended to analyze the actions carried out in the European and national territory.

\section{KEY WORDS:}

Communication - Europe - Spain - Sustainability - Sustainable innovation - Tourism.

\section{SETOR TURISMO: COMUNICAÇÃO E INOVAÇÃO SUSTENTÁVEL}

\section{RESUMO}

Este artigo é desenvolvido a partir da premissa de analisar os meios e ferramentas implementados em Espanha para a melhoria e condicionamento do setor turístico no que diz respeito à sustentabilidade. São analisados os motivos que levaram a indústria hoteleira a implementar medidas de mudança para a preservação do meio ambiente e do patrimônio cultural espanhol. Pretende-se também analisar as ações realizadas em território europeu e nacional.

\section{Cómo citar el artículo:}

Barrientos-Báez, A., Caldevilla Domínguez, D., Cáceres Vizcaíno, A. y Sueia Val, E. G. (2020). Sector Turístico: Comunicación e Innovación sostenible [Tourism Sector: Communication and Sustainable Innovation]. Revista de Comunicación de la SEECI, $53,153-173$

doi: https://doi.org/10.15198/seeci.2020.53.153-173

Recuperado de http://www.seeci.net/revista/index.php/seeci/article/view/680

\section{INTRODUCCIÓN}

Nos planteamos conceptualizar una palabra que desde hace una década se escucha en medios de comunicación, círculos empresariales e institucionales: sostenibilidad. Se "busca garantizar las necesidades del presente sin comprometer a las futuras generaciones. Se basa en tres pilares fundamentales: el desarrollo social, la protección del medio ambiente y el crecimiento económico" (Naciones Unidas, 1987).

Asimismo, podemos admitir que el tema expuesto merece una investigación debido a la importancia que actualmente ocupa el sector turístico en la sociedad, y cómo está afectando al medio ambiente. Tal y como se describe, "el concepto de sostenibilidad surge por una vía negativa, como resultado de los análisis de la situación del mundo, que puede describirse como una "emergencia planetaria" (Bybee, 1991), "como una situación insostenible que amenaza gravemente el futuro de la humanidad" (Macedo, 2005). Hay que mencionar también que, el pasado 21 de enero de 2020 el Gobierno 
de España declaró la emergencia climática y ambiental que desde hace tiempo la comunidad científica viene avisando de su gran magnitud (Gobierno de España, 2020).

En relación al sector turístico, éste es también culpable de que la situación del planeta se encuentre en tan mal estado. Quintero (2004) admite que "el crecimiento incontrolado de un turismo destinado a obtener beneficios a corto plazo tiene a menudo consecuencias negativas, puesto que daña el medio ambiente y las sociedades y destruye las mismas bases sobre las que el turismo se asienta y desarrolla". Es por ello que, el turismo de masas, la sobreexplotación de los destinos y de los recursos naturales, además de la huella de carbono generada por los desplazamientos aéreos son algunos de los factores que han generado esta situación de crisis ambiental, además de todas las industrias que participan para que este sector genere su ciclo económico.

Es primordial investigar sobre este tema para el avance y posterior mejora de la industria del turismo y de las consecuencias que ésta produce. El desarrollo turístico tradicional en España, ha generado un modelo de crecimiento extensivo basado en el incremento continuado del número de visitantes. Tal es así que hay estudios que aseguran que las relaciones públicas en España principian con la necesidad del desarrollo del turismo y la mejora de la imagen del país a través de él (Caldevilla Domínguez et ál., 2020).

La prioridad de los beneficios a corto plazo, la creación de una oferta homogénea y estandarizada, la escasa presencia de la cultura y las tradiciones locales en la configuración de esta oferta y, en general, una fuerte presión ambiental, que ha provocado graves impactos en la calidad ambiental y paisajística del territorio, han conformado un modelo, en esencia insostenible, que perdura desde hace cuatro décadas.

Se tratará de desarrollar argumentos que vinculan al turismo con una vía sostenible para el desarrollo y conservación del entorno (plano físico) y de las personas (plano mental), con miras a un futuro a largo plazo donde se apueste por la implementación de medidas reales y alcanzables que promuevan el uso de energías renovables y la inclusión de la sociedad local principalmente. Revisaremos las medidas tomadas en España comparándolas con las practicadas en otros países respecto a la funcionalidad del turismo desde la perspectiva del desarrollo sostenible de donde podemos concluir, al igual que Barrientos-Báez et ál. (2019), que es preciso un cambio en los paradigmas educativos para lograr que los de turismo se encaucen hacia la sostenibilidad e imbricación medio-persona, incluyendo la modificación de los planes de estudio. También es cierto que queda camino por andar en otros muchos campos de la educación superior en lo referente a las competencias sociales (Sánchez-Marín et ál., 2019). Una nueva educación ha de ser la base para poder hacer gravitar el peso de la misma en torno a estos nuevos contenidos.

En cuanto al desarrollo de la investigación, se ha realizado una búsqueda de información en fuentes bibliográficas recientes, artículos científicos e información 
publicada en diferentes medios de comunicación. A continuación, se establecen unos objetivos para marcar un camino propio en la investigación, y en el marco teórico se conceptualizan los conceptos claves del proyecto. A su vez, se llevará a cabo un análisis de las medidas sostenibles que actualmente se llevan a cabo en Europa y en España, para poder determinar si son eficaces y además, tomarlos de ejemplo para una plausible aplicación en los destinos turísticos españoles. La comunicación con emoción puede representar una guía de valores y comportamientos (Carretero-Díaz y Barrientos-Báez, 2019).

Es importante destacar que, para que este proyecto se desarrolle con éxito es primordial la participación y colaboración de todos los organismos relacionados con el sector. Además de la regulación de leyes actuales, entre alguna de ellas, el Real Decreto 416/2014, de 6 de junio, por el que se aprueba el Plan sectorial de Turismo de Naturaleza y Biodiversidad 2014-2020, que tiene como objetivo la adecuada conservación y gestión sostenible de la biodiversidad y con ello, acentuar la integración de las políticas sectoriales consiguiendo reforzar los vínculos existentes entre la conservación del medio natural y el desarrollo económico y social (BOE, 2014). Por otro lado, es importante tener en cuenta la capacidad de carga turística de los destinos y la huella ecológica, e implementar el uso de indicadores que garanticen la sostenibilidad de los servicios turísticos.

En consecuencia, el presente trabajo pretende profundizar en el óptimo conocimiento de las medidas o acciones sostenibles actuales en el sector turístico para potenciarlas, y así minimizar los impactos negativos que genera el turismo en el medio, preservando el medio ambiente, el patrimonio cultural, material e inmaterial (Walls, 2020). Por otro lado, gracias al proceso de investigación se analizarán nuevas formas de fomentar recursos tanto naturales como humanos. Más aún, establecer formas de favorecer y apostar por los comercios locales, e incluir a los españoles como importantes puntos de apoyo para la conservación y mantenimiento del territorio.

\section{OBJETIVOS}

"El objetivo de la nueva Estrategia de Turismo Sostenible de España 2030 es sentar las bases de la transformación del turismo español hacia un modelo de crecimiento sostenido y sostenible, que nos permita mantener su posición de liderazgo mundial. El nuevo modelo, estará apoyado en la mejora de la capacidad competitiva y rentabilidad de la industria, en los valores naturales y culturales diferenciales de los destinos, y en la distribución equitativa de los beneficios y las cargas del turismo". (Secretaria de Estado y Turismo, 2019)

Considerando que los objetivos del presente artículo parten de la premisa anteriormente mencionada, nos planteamos:

1) Conocer la realidad del sector en cuanto a sostenibilidad turística.

2) Analizar los motivos que han llevado a la industria hotelera a implementar medidas.

3) Desarrollar posibles medidas de cambio en el mercado turístico que incluyan: 
- El desarrollo equilibrado del turismo en el territorio.

- Impulsar la sostenibilidad como valor de marca del turismo en España.

\section{METODOLOGÍA}

Este trabajo se ha realizado con el fin de investigar y conocer la importancia que tiene en la actualidad la sostenibilidad y las herramientas que se implementan en el sector turístico para un correcto y generoso uso para la sociedad en su conjunto y para la industria turística en particular.

Se ha comenzado por una etapa cualitativa, a partir de una revisión bibliográfica y una búsqueda activa de información sobre los temas significativos y adyacentes a los factores clave que definen la sostenibilidad y el cuidado de todo aquello que nos rodea. Se han identificado los elementos y trabajos referidos al área de estudio para compartirla mayormente en el epígrafe Discusión, donde podremos encontrar los conceptos e información relevante referida a nuestro campo de estudio.

\section{SOSTENIBILIDAD Y TURISMO}

\subsection{Concepto de Sostenibilidad}

En la actualidad, uno de los temas relevantes para la humanidad en el desarrollo de la sociedad y en sus diferentes determinaciones (ambientales, económicas y sociales) sigue siendo el relacionado con la sustentabilidad y/o sostenibilidad de la misma (Zarta, 2018). Desde que se realizó la conferencia de las Naciones Unidas para el Desarrollo y la Sostenibilidad en Río de Janeiro en 1992, el principio fundamental de la sostenibilidad es bien conocido en el discurso científico y político internacional (Menzel, 2001).

La base lingüística del concepto de sostenibilidad tiene su origen en el latín, y proviene de la palabra sustenere: sostener, sustentar, soportar. Ahora bien, si se plantea el origen de este término es de gran asombro saber que se remonta en el concepto "Nachhaltigkeit" del jurista alemán Hans Carlowitz (1645-1714) quien desarrolló en 1713 una teoría sobre la utilización óptima de los bosques, que fueron las fuentes de energía para la protoindustria de hierro y plata. Planteó que el volumen de la producción de esta industria no podía ser superior a la velocidad de reproducción de los bosques (Marquardt, 2006). Esto es por consiguiente, una clara ejemplificación de lo que la sostenibilidad trata de regular.

Para comprender más allá la teoría de Carlowitz debemos partir de la idea básica, y es que una sociedad no debe usar más recursos de los que renueva, para que la siguiente generación pueda tener las mismas oportunidades de acceso a dichos recursos. El límite de uso de estos últimos para el desarrollo de las actividades de las sociedades y de sus economías depende de la capacidad y reproductividad intergeneracional de los ecosistemas (Marquardt, 2006). 
Se podría afirmar que el término de la sostenibilidad se ha redescubierto, desde la década de los noventa forma parte del derecho internacional y constitucional, y se le considera como el programa del futuro desarrollo global. Contiene cuatro subprincipios como finalidades del Estado y mandatos a la legislación (Marquardt, 2003):

1. El principio de la regeneración entendido como el adaptarse a los ciclos naturales de los recursos renovables.

2. El principio de la sustitución de recursos no renovables, como petróleo y uranio.

3. La perspectiva integrada de la economía, la sociedad y la ecología.

4. El principio mundial entendido como el equilibrio planetario que no debe exceder la capacidad de carga global.

A pesar de que el concepto de sostenibilidad es sencillo, hay que mencionar que a lo largo de su historia ha generado cierta controversia, y que según su interés puede derivar en unas u otras características. En general, lo que interesa sostener a unos no tiene por qué coincidir con lo que interesa sostener a otros y el conflicto está servido. Pero la conciencia del conflicto es también el punto de partida para su resolución. Además de asumir que la sostenibilidad no hace frente a un único problema, sino a un buen número de ellos (González y Menéndez, 2008). Simultáneamente, avanzar hacia la sostenibilidad exige cambiar la forma de pensar, de actuar, de producir y de consumir. En este proceso hacia la sostenibilidad son actores clave las autoridades, las empresas, las organizaciones sociales y los individuos (Fernández, 2015).

En síntesis, la definición de "sostenible" involucra diversos aspectos de gran importancia, entre los cuales podemos contemplar (Zarta, 2018):

$\rightarrow$ La sustentabilidad tiene que ver con lo finito y limitado del planeta, así como con la escasez de los recursos de la tierra.

$\rightarrow$ Con el crecimiento exponencial de su población.

$\rightarrow$ Con la producción limpia, tanto de la industria como de la agricultura.

$\rightarrow$ Con la contaminación y el agotamiento de los recursos naturales.

\subsection{Concepto de Desarrollo Sostenible}

El antecedente del término desarrollo sostenible es el concepto de ecodesarrollo, defendido por Strong en 1973, primer director ejecutivo del PNUMA (Programa de las Naciones Unidas para el Medio Ambiente): "Es el desarrollo a nivel regional o local, congruente con las potencialidades del área en cuestión, prestando atención al uso adecuado y racional de los recursos naturales y a la aplicación de estilos tecnológicos apropiados, y a la adopción de formas de respeto hacia los ecosistemas naturales, centrando su objetivo en utilizar los recursos según las necesidades humanas, mejorando y manteniendo la calidad de la vida para esta generación y las futuras".

El concepto tiene su origen en la Comisión Mundial sobre Medio Ambiente y Desarrollo, de las Naciones Unidas celebrado en 1987, definido en el Informe Brundtland de la siguiente forma: "Desarrollo sostenible es el desarrollo que asegura 
las necesidades del presente sin comprometer la capacidad de las generaciones futuras para asumir sus propias necesidades" (Comisión Mundial del Medio Ambiente y del Desarrollo, 1988), y tiene además las siguientes características: mantiene la calidad de vida general, permite un acceso continuado a los recursos naturales e impide que perduren los daños al medio ambiente.

Fernández (2015, p. 11) señala que, "el desarrollo sostenible requiere cubrir las necesidades básicas para todos y la extensión de satisfacer sus aspiraciones a una vida mejor. Pero también requiere la promoción de valores que estimulen estándares de consumo que estén entre los límites de lo ecológicamente posible y a los que todos pueden razonablemente aspirar. Simultáneamente, el desarrollo sostenible acarrea una transformación paulatina de la sociedad y de la economía, además de recalcar la necesidad de que este desarrollo se dé en un marco político y social adecuado".

Por otro lado, el concepto de desarrollo sostenible (Altaraz, 2002) "no sólo se ha ceñido a las discusiones sobre el capital natural y humano, sino que ha ido más allá. El concepto ha incorporado aspectos económicos, sociales y ambientales, participando en este sentido todos los sectores de la sociedad en la toma de decisiones". Según algunas de las interpretaciones de diferentes autores, el desarrollo sostenible consistirá en:

1. Sostener los recursos naturales (Carpenter, 1991).

2. Sostener los niveles de consumo (Redclift, 1987), hace referencia a los modelos de consumo de los países ricos que impiden el desarrollo sostenible a nivel mundial.

3. Lograr la sostenibilidad de todos los recursos: capital humano, capital físico, recursos ambientales, recursos agotables (Bojo et ál., 1990).

4. Perseguir la integridad de los procesos, ciclos y ritmos de la naturaleza (Shiva, 1989).

5. Sostener los niveles de producción. "Una actividad sostenible es aquella que o bien utiliza productos renovables o bien, al menos, financia la puesta en marcha de un proyecto que asegure tanto la producción renovable de un bien sustitutivo del consumidor, como la absorción de residuos generados sin daño para los ecosistemas" (Naredo, 1990).

El Informe Brundtland (1987) considera que "el desarrollo económico, el desarrollo social y la protección medioambiental son componentes interdependientes del desarrollo sostenible que se refuerzan mutuamente". En el Consejo Europeo de Gotemburgo, Nicole Fontaine, Presidenta del Parlamento Europeo de 2001 recalcó: "la voluntad de la Unión Europea a favor de un desarrollo sostenible, cuyas tres dimensiones, la económica, la social y la medioambiental, son indisociables".

En pocas palabras y para concluir el término, el desarrollo sostenible ha sido creado como un modelo alternativo que tiene en cuenta todos los ámbitos necesarios para garantizar un futuro próspero para las siguientes generaciones. Actualmente, el compromiso establecido por las Naciones Unidas está dando buenos resultados, gracias al establecimiento de los 17 Objetivos de Desarrollo Sostenible que ha 
proporcionado un modelo para la prosperidad compartida en un mundo sostenible: un mundo en el que todas las personas puedan llevar una vida productiva, vibrante y pacífica en un planeta sano.

\subsection{Concepto de Turismo Sostenible}

Tras la divulgación de los términos "sostenibilidad" y "desarrollo sostenible", se considera necesario, según Wall y Mathieson (2006), "describir un desarrollo ideal del turismo que no impliquen impactos ambientales y sociales negativos". El turismo sostenible es un concepto que empieza a generar interés desde la perspectiva de crecimiento y desarrollo económico, manteniendo el equilibrio entre producción, recursos y naturaleza (Murcia et ál., 2017). En concreto, La Organización Mundial del Turismo (OMT) considera que: "El desarrollo del turismo sostenible responde a las necesidades de los turistas y de las regiones anfitrionas presentes, a la vez que protege y mejora las oportunidades del futuro. Está enfocado hacia la gestión de todos los recursos de manera que satisfagan todas las necesidades económicas, sociales y estéticas, y a la vez que respeten la integridad cultural, los procesos ecológicos esenciales, la diversidad biológica y los sistemas de soporte de la vida" (Informe Brundtland, 1987).

Sin embargo, a pesar de la determinación del concepto por parte de la OMT, las definiciones de "turismo sostenible" son diversas, por lo que no queda claro cuál es el criterio general sobre su interpretación, posiciones o alcance para hacerlo efectivo. Hecha esta salvedad, Bertoni (2008) afirma que "es necesaria una aproximación conceptual del turismo sostenible que permita salvar la vaguedad del término y su incorrecta apropiación para un desarrollo operativo". Conforme a esto, a continuación se abordan las características principales y más importantes para clarificar el concepto del "Turismo Sostenible". En suma, es primordial saber que este concepto pretende aplicar los principios de desarrollo sostenible a la actividad turística, ya que como Fernández (2015) señala, "el turismo integrado en el desarrollo sostenible conserva y recupera valores culturales, sociales y medioambientales, porque la sostenibilidad global solo puede ser alcanzada por comunidades regionales y estatales sostenibles". De igual manera, la aplicación de los principios de la sostenibilidad al turismo es una referencia esencial en los procesos de desarrollo y promoción de los destinos potenciales y de reestructuración de los destinos actuales (Bertoni, 2008).

Ahora bien, tras haber analizado artículos especializados, éstos presentan en común varios elementos característicos del turismo sostenible:

1) Debe existir una armonía entre las aristas economía, naturaleza y sociedad (Cardoso et ál., 2014).

2) Fomenta respetar la cultura, tradiciones, naturaleza y patrimonio local (Cañizares, 2013).

3) Los ingresos derivados de la actividad turística deben revertirse hacia el desarrollo local (Ortiz, 2010).

4) Debe existir un trabajo sistémico entre los actores involucrados en la actividad turística y debe considerarse como un modelo viable de desarrollo, que 
garantice las necesidades de las presentes y futuras generaciones (Orozco, 2013).

Al mismo tiempo, se considera fundamental para el posible alcance de la actividad turística desde la perspectiva sostenible que "el desarrollo sostenible del turismo requiere de la participación de todas las partes interesadas, así como un fuerte liderazgo político para garantizar una amplia participación y consenso" (OMT, 2005).

Por otro lado, el turismo sostenible debe mantener el nivel de satisfacción de los turistas para asegurar experiencias significativas, además de sensibilizar sobre temas medioambientales y promover prácticas de turismo sostenible entre ellos. Además, es preciso señalar que el turismo sostenible no es un producto, sino una base filosófica necesaria para apoyar y proporcionar una orientación para desarrollar el turismo en los destinos y hacer buen uso de los recursos naturales y culturales que en este se albergan (Cardoso et ál., 2014), esta forma es la que los turistas deben concebir la imagen de turismo sostenible.

Finalmente, el término "Turismo Sostenible" se sintetiza como un proceso de cambio cualitativo producto de la voluntad política que, con la participación imprescindible de la población local, adapta el marco institucional y legal, así como los instrumentos de planificación y gestión, desarrollo turístico basado en el equilibrio entre la preservación del patrimonio natural y cultural, la viabilidad económica del turismo y la equidad social del desarrollo (Ivars, 2001, p. 11).

El turismo integrado en el desarrollo sostenible no sólo conserva y recupera valores culturales, sociales y medioambientales, porque la sostenibilidad global sólo puede ser alcanzada por comunidades regionales y estatales sostenibles, sino que realza nichos culturales y puntos de atracción para públicos concretos (Gallego Jiménez y Vidal Raméntol, 2018) o se basa la nueva comunicación publicitaria en reflejar los tratamientos inclusivos de dichos públicos (Lorite García et ál., 2018). Avanzar hacia la sostenibilidad exige cambiar de forma de pensar, de actuar, de producir y de consumir y ampliar mercados desde su propia especificidad.

\subsection{Sostenibilidad Turística en Europa}

En la década de los años sesenta se origina la preocupación a nivel internacional por el tema del medio ambiente, como consecuencia surge el movimiento ecologista y/o ambientalista contemporáneo, proponiendo cambios importantes a nivel político, social, cultural y económico (Zarta, 2018). Asimismo, desde la aceptación del concepto Desarrollo Sostenible y a su vez Turismo Sostenible, son algunas de las instituciones internacionales las que apoyan y asumen este concepto, entre ellas se encuentran: la Organización de Naciones Unidas para la educación y la cultura (UNESCO), El programa de Naciones Unidas para los países menos adelantados (PNUMA), La Organización Mundial del turismo (OMT) y la Unión Europea (UE) (Ávila, 2002).

Esta última, específicamente en la Unión Europea, se ha ido desarrollando el marco político y regulatorio medioambiental más ambicioso del mundo. La diplomacia 
climática y medioambiental europea posee un papel fundamental, liderando proyectos primordiales, como los Objetivos de Desarrollo Sostenible o el Acuerdo de París (Calleja y Alquézar, 2020).

Un hecho significativo tuvo lugar en el año 1999, cuando La Organización Mundial del Turismo (OMT) solicitó a las administraciones nacionales de turismo que sus estados enviarán diferentes propuestas de políticas, turísticas, iniciativas o proyectos que fueran consideradas sostenibles. Recibieron casi cincuenta casos, de las cuales las mayorías hablaban sobre el ecoturismo o el turismo rural, actividades especialmente afines a la sostenibilidad, pero también se llegó a la conclusión del poco conocimiento que existía del concepto de turismo sostenible, ya que este debe aplicarse a todo tipo de actividades turísticas. Es por ello que se considera importante que, este concepto no se aplique solo a productos minoritarios, si no que sirva como desafío global de transformación, que se aplique a los destinos masivos, a la gran industria del turismo, a los viajes, desde el transporte hasta el alojamiento (Ávila, 2002).

Como afirma la política medioambiental europea, no solo procura proteger la naturaleza, si no que pretende ejercer un papel fundamental para mantener la competitividad y crear puestos de trabajo. Así pues, tenemos que reforzar nuestras credenciales verdes y nuestra capacidad de innovación sostenible, mediante la eficiencia energética y de los recursos, o de la economía circular (Calleja y Alquézar, 2020). A continuación, mediante la elaboración de esta investigación, se establece una serie de medidas sostenibles, las cuales hemos considerado más relevantes, implementadas en la Unión Europea, como refuerzo a la sostenibilidad en la industria turística. (Ver figura 1) 
-Federación EURoparC

-Conferencia de las Naciones Unidas sobre el Medio Humano.

-Informe Brundtland: "Nuestro Futuro Común"

-Conferencia de las Naciones Unidas sobre Medioambiente y Desarrollo (CNUMAD)

- La Cumbre del Milenio

- La Cumbre de Lisboa

-El Libro Verde

-Europa, primer destino turistico mundial: Un nuevo marco político para el turismo europeo" COM 352

-RIO+20 Conferencia de Desarrollo Sostenible: Se inician los ODS

-Sistema Europeo de Indicadores Turísticos (ETIS)

Estrategia europea para un mayor crecimiento y empleo en el turismo costero y marítimo (COM)

-Programa Marco de la Unión Europea, Horizonte 2020

-Acuerdo de París. Agenda 2030: Objetivos de Desarrollo Sostenible

- Medición de la Sostenibilidad Turística (MST)

-Plan de Acción para la Economía Circular

Figura 1. Medidas Sostenibles Aplicadas en la Unión Europea.

Fuente: Recopilación del Ministerio de Turismo. Ver apartado de referencias.

En la actualidad, en el contexto de la Agenda 2030 para el Desarrollo Sostenible y de los Objetivos de Desarrollo Sostenible (ODS), se establecen los 17 objetivos de desarrollo sostenible elaborados por las Naciones Unidas y propuestos por el Grupo de Trabajo Abierto de la Asamblea General (ONU, 2015). En cuanto al sector turístico podemos relacionarlo directamente con tres de ellos, específicamente en los objetivos 8,12 y 14 , los cuales hacen hincapié sobre el trabajo decente y crecimiento económico, además, de la promoción y el consumo responsable y por último, la sostenibilidad de los océanos, respectivamente. Sin embargo, el turismo puede contribuir, directa e indirectamente a los 17 objetivos en vista de su naturaleza transversal y, a su vez, estimular el crecimiento económico inclusivo, crear empleos, atraer inversión, combatir la pobreza, mejorar el desarrollo de las comunidades locales, promover el empoderamiento de mujeres y jóvenes, proteger el patrimonio cultural, preservar la tierra y el mar ecosistemas y biodiversidad y finalmente apoyar la lucha contra el cambio climático (UNWTO, 2018). 
Cabe destacar que Naciones Unidas declaró el 2017 como Año Internacional del Turismo Sostenible para el Desarrollo. Es por ello que el Año Internacional investiga y acentúa el papel del turismo en los siguientes términos (OMT, 2016):

1. Crecimiento económico inclusivo y sostenible

2. Inclusión social, empleo y reducción de la pobreza

3. Uso eficiente de los recursos, protección ambiental y lucha contra el cambio climático

4. Valores culturales, diversidad y patrimonio

5. Comprensión mutua, paz y seguridad"

\subsection{Sostenibilidad Turística en España}

El desarrollo turístico en España ha generado un modelo de crecimiento basado en el incremento continuado del número de visitantes sin prestar atención a la capacidad de carga del territorio (Fernández, 2020). Asimismo, el boom turístico de los años 50 supuso un alto nivel de ingresos y popularidad, pero también serios perjuicios en el entorno cuando todavía no se prestaba atención a la sostenibilidad turística (Plumed et ál., 2018).

España, como destino popular de sol y playa acarreó una construcción masiva no planificada, sin pensar en el impacto medioambiental. A todo ello hay que sumar que la densidad de población de las localidades costeras españolas es cinco veces mayor que la media nacional y se triplica durante la temporada alta (Vogeler y Hernández, 2003), siendo el impacto poblacional uno de los aspectos más importantes a considerar en el desarrollo sostenible (Castrillón et ál., 2015).

La prioridad en aquel momento no solo eran los beneficios a corto plazo y la creación de una oferta homogénea y estandarizada sino que, esta oferta presentaba escasa presencia de la cultura y las tradiciones locales, además de una fuerte presión ambiental y paisajística del territorio. Se trató de un nuevo modelo insostenible que ha perdurado a lo largo de más de cuatro décadas (Ayuso, 2003).

España es una de las principales potencias del mundo en este sector. Desde la perspectiva económica, el desarrollo de las actividades turísticas tiene efectos muy positivos en nuestro país, con una aportación importante al valor añadido bruto y al saldo de la balanza por cuenta corriente. En el año 2018, España recibió 82,6 millones de turistas internacionales, cifra récord, lo que la sitúa en el segundo país del mundo que más visitantes extranjeros recibe, por detrás de Francia (Fernández, 2020).

Sin embargo, a pesar de la gran experiencia turística con la que cuenta España no significa que la tenga en el ámbito del turismo sostenible. Fernández (2020) advierte, "hay también una serie de efectos perversos, negativos sobre el medio ambiente y en especial sobre los recursos turísticos naturales, que hay que tener en cuenta. En efecto, los impactos más relevantes coinciden con las zonas explotadas, como, la presión urbanística, el consumo de recursos naturales y otros elementos de presión ambiental, que convierten a grandes zonas de atracción turística en lugares en los 
que se hace cada vez más necesaria la implantación de estrategias de desarrollo sostenible, y la adaptación de medidas de política económica".

Asimismo, como destino turístico líder, España debe incorporar la sostenibilidad a la planificación turística.

Los Ministerios implicados indicaron que los destinos deben proteger sus recursos naturales para ser competitivos a largo plazo (Ministerio de Economía y Hacienda, y Ministerio de Medio Ambiente, 1999), considerando el respeto a la población local y a los territorios. Cuando el Gobierno español comprendió la importancia de la gestión sostenible del turismo, los planes políticos empezaron a cambiar su percepción de la actividad turística y la influencia de la sostenibilidad en el desarrollo del turismo fue obteniendo un papel cada vez más fundamental (Plumed et ál., 2018).

Ahora bien, tras haber realizado una investigación sobre las medidas incorporadas por el Gobierno español se ha realizado un listado de las consideradas más relevantes, las cuales según Cañizares Ruiz (2013) "han condicionado un nuevo enfoque del turismo acorde con las exigencias de un modelo de desarrollo más sostenible". (Ver Figura 2)

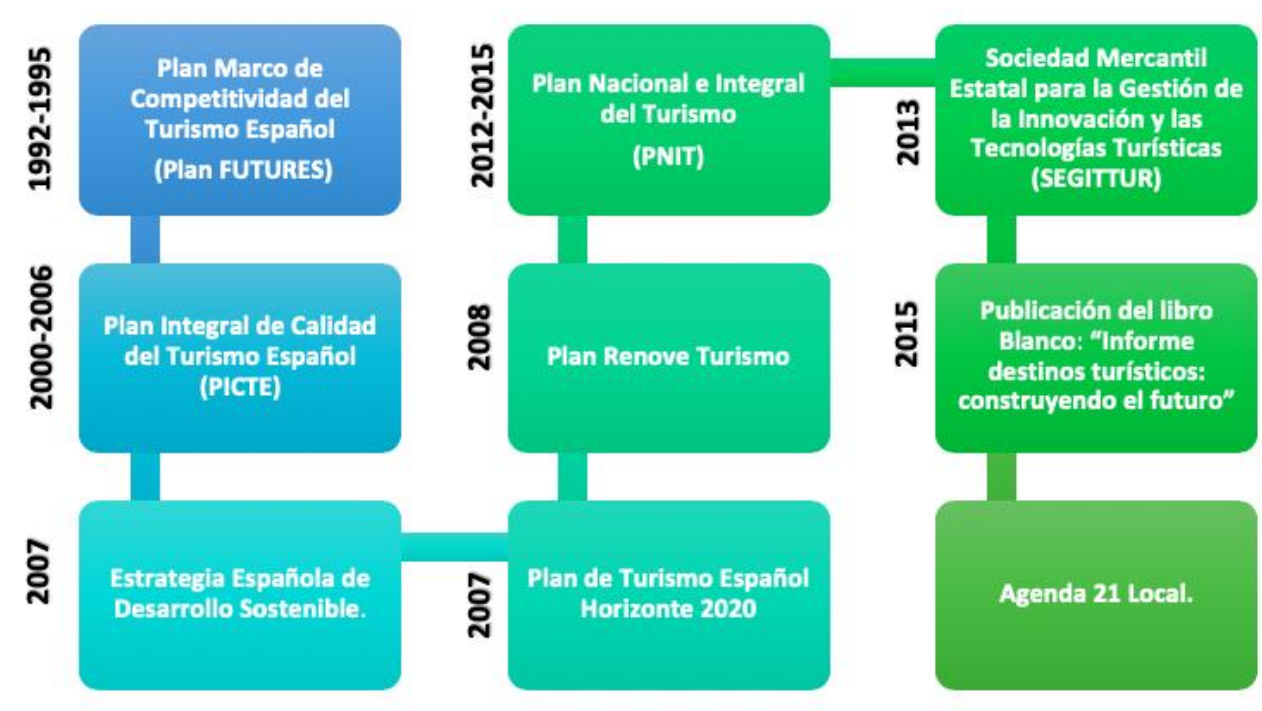

Figura 2. Medidas implementadas en España

Fuentes: Recopilación del Ministerio de Turismo. Ver apartado de referencias.

Es preciso mencionar que, la aparición de una creciente y potente competencia, la penetración y extensión de las nuevas tecnologías e internet, y los nuevos modelos de transporte han sido tres aspectos claves de la revolución que ha vivido en los últimos 20 años el sector turístico en España y en todo el mundo (Zoreda y Perelli, 2014). Más aún, con el objetivo de concluir con este epígrafe, se considera relevante exponer los casos de dos ciudades turísticas españolas que son un claro ejemplo de que la incorporación de medidas sostenibles en el sector turístico es posible. 
Por lo que se refiere, la ciudad de Benidorm (Alicante) es uno de los destinos más emblemáticos del panorama turístico español. Esta ciudad nació bajo el modelo fordista y los paquetes turísticos de los años 1950, y se convirtió en un destino de sol y playa caracterizado por el turismo de masas. Sin embargo, en los últimos años se ha creado el proyecto "Benidorm, destino turístico inteligente y sostenible", el cual ha transformado y consolidado el ayuntamiento como Destino Turístico Inteligente. Mediante el uso de la innovación y la tecnología se pretende alcanzar un destino completamente accesible y que garantice la sostenibilidad tanto económica, como sociocultural y medioambiental. Asimismo, en lo que a la sostenibilidad respecta, Benidorm cuenta con la recopilación de información sobre la concentración de partículas de óxidos de nitrógeno y ozono, sobre ocupación hotelera y cuál es el impacto en la comunidad local (Martín y Sánchez, s.f.). Esta nueva imagen que se pretende actualizada a las nuevas exigencias de los tiempos ha de ir acompañado indefectiblemente de un nuevo concepto de RSC en el sector hostelero (Castillo y Villagra, 2019).

Una de las medidas aplicadas es la siguiente: a los indicadores tradicionales se le asignó otras mediciones para otorgarles un grado significativo de importancia. Por ejemplo, a los elementos del patrimonio cultural se les ha atribuido algún tipo de protección oficial o plan de conservación. Acorde con las nuevas medidas incorporadas, en el año 2019, Benidorm ha sido el primer territorio en recibir la certificación de Destino Turístico Inteligente (DTI) en base a la normativa UNE 178501 de AENOR.

Sin embargo, el Informe de IDITUR (Centro de Investigación, Divulgación e Innovación Turística) explica que también otros destinos nacionales han realizado avances en los cuatro ejes que componen un DTI. Es el caso de Lloret de Mar (Girona).

Conviene destacar que Lloret de Mar a pesar de ser un destino maduro de sol y playa ha apostado por impulsar la implementación de las tecnologías de la información y las comunicaciones en el funcionamiento de las entidades turísticas. El ayuntamiento de esta localidad, la Mesa Empresarial de Turismo y la Generalitat han tomado como premisa el Plan Estratégico de Turismo de Lloret de Mar 2010-2014, además de la colaboración del Plan Operativo de Renovación del Destino Turístico: Lloret De Mar 2015-2020. Con este plan, se propone ofrecer productos y servicios competitivos y contribuir a un modelo de destino que favorezca la sostenibilidad, la accesibilidad y el bienestar de residentes y turistas (Martín y Sánchez. s.f).

Cabe destacar además que, las estrategias en materia de sostenibilidad y políticas ambientales que se llevan a cabo desde el Ayuntamiento se pueden dividir en cuatro grandes bloques: residuos, ciclo del agua, aire y energía, y entorno natural. En cuanto las políticas ambientales, desde el consistorio se está trabajando en proyectos que ponen en valor el entorno natural y su mantenimiento, y también en estrategias de política turística como el turismo deportivo, de congresos y reuniones, o el turismo familiar, que ayudan a desestacionalizar la temporada y por tanto, evitar la presión que se ejerce sobre el territorio en épocas de máxima afluencia estival (Lloret de Mar trabaja para posicionarse como un destino sostenible, 2017). 


\section{CONCLUSIONES}

La industria turística se está enfrentando a grandes cambios. Los problemas ambientales actuales son de carácter urgente y afectan no solo al sector turístico, sino a la sociedad en todos sus ámbitos. Los límites de crecimiento se han sobrepasado y las consecuencias se reflejan principalmente en el cambio climático.

Se trata de un problema que concierne a toda la humanidad. Sin embargo, se considera que el cambio debe comenzar por pequeñas iniciativas en las que demostrar que es posible y alcanzable. Por ello, este artículo se ha enfocado en desarrollar medidas sostenibles para innovar el sector turístico español y promover el cuidado y mantenimiento del planeta.

Cabe mencionar, que en el desarrollo del proyecto se han encontrado limitaciones debido a la situación recientemente vivida por el COVID-19, por lo que no ha sido posible desarrollar todos los planes iniciales que buscaban indagar en profundidad la situación con entes de prestigio para el desarrollo sostenible. Por otro lado, la cuantía de artículos e investigaciones halladas han sido numerosos, por ello, se ha tenido que acotar el número de páginas añadiendo anexos.

Primordialmente, el análisis de la actualidad turística demuestra que desde hace más de una década, los expertos vienen informando de la urgencia de implementar nuevas formas de realizar turismo, y a pesar de los avances realizados por las políticas turísticas, en donde se ha apostado por medidas innovadoras y sostenibles, los países receptores de turismo han optado por el beneficio económico sin pensar en el impacto que generaban en el medio. En consecuencia, es necesario seguir invirtiendo en investigar y trabajar por aplicar estos cambios con carácter inminente.

No obstante, la responsabilidad de aplicar una visión sostenible no solo es de los estados, sino también de la sociedad. Ahora bien, es de gran gratitud revelar que recientes estudios demuestran que gran parte de la población muestra interés y preocupación, además de presentarse voluntarios ante la necesidad de un cambio para mejorar la situación turística en Tenerife.

Aprovechando la situación estratégica de España y con las medidas propuestas como plan de acción, es clave persistir en la concienciación y educación social, para convertir a España en un conjunto de potenciales destinos turísticos sostenibles como referente a nivel internacional. Véanse casos similares en política organizacional sostenible como los textos de Salvatierra Iriarte (2018) o RodríguezFernández y Vázquez-Sande (2019) que ayudan a perfilar las políticas de futuro al respecto partiendo de la necesaria actualización de los modelos de gestión en vigor y anteriores.

La base para el progreso se halla en la movilidad y en la ayuda humanitaria por la obtención de un futuro común y próspero para todos los seres que habitan el planeta. 
Como apunta Barrientos-Báez (2016), contamos con unas herramientas increíbles que permiten que los procesos de aprendizaje sean mucho más personalizados, mucho más flexibles. En nuestro caso, la sostenibilidad y el respeto por el medio ambiente podrían considerarse como conceptos prioritarios en el aprendizaje y formación de los jóvenes.

\section{REFERENCIAS}

Altaraz, M. (2002). Teoría de las tres dimensiones de desarrollo sostenible. Ecosistema: Revista de Ecología y Medioambiente, 11(2). https://bit.ly/2yWtM46

Andrade, S. (2020). ¿Será el Hierro la primera isla española en ser $100 \%$ sostenible? Traveler. https://bit.ly/2AjMJxY

AVA-Q. (2016). Nuestras propuestas para un Turismo Sostenible. Alternativa Verde por Asturias. https://bit.ly/3gunFFf

Ávila, R. (2002). Turismo Sostenible. IEPALA Editorial. ISBN: 84-89743-20-7. https://bit.ly/2AoAd0r

Ayuso, S. (2003). Gestión sostenible en la industria turística. Retórica y práctica en el sector hotelero español [Tesis Doctoral, Universitat Autónoma de Barcelona] ISBN: 8468860646. http://hdl.handle.net/10803/4954

Azcárate T., Benayas J., Nerilli G. y Justel A. (2019). Guía para un turismo sostenible: Retos del sector turístico ante la Agenda 2030. https://bit.ly/2WsAKHb

Barrientos-Báez, A. (2016). GDS Amadeus. Propuesta de innovación didáctica. Capítulo 3. TIC actualizadas para una nueva docencia universitaria. McGraw Hill.

Barrientos-Báez, A., Barquero-Cabrero, M. y Rodríguez-Terceño, J. (2019). La educación emocional como contenido transversal para una nueva política educativa: el caso del grado de turismo. Revista Utopía y Praxis Latinoamericana. 24(4), 147-165

Bertoni, M. (2008). Turismo sostenible: su interpretación y alcance operativo. Cuadernos de Geografía: Revista Colombiana de Geografía, (17), 155-163. https://bit.ly/3cGetLT

Bojo, J., Maler, K. y Unemo, L. (1990) Environment and development: an economic approach. Springer-Science+Business Media, B.V. https://bit.ly/2YZImUI

Bybee, R. (1991). Planet Earth in crisis: How should science educators respond? The American Biology Teacher, 53(3), 146-153. https://bit.ly/3dLrsff

Caldevilla-Domínguez, D., Barrientos-Báez, A. y Fombona-Cadavieco, J. (2020). Evolución de las Relaciones Públicas en España. El Profesional de la Información, 29(3), https://doi.org/10.3145/epi.2020.may.05 
Calleja, D. y Alquézar, J. (2020). Desarrollo Sostenible y competitividad: La visión de la Unión Europea. ICE: Revista De Economía, 912, 15-27. ISSN 0019-977X. 10.32796/ice.2020.912.6962

Cañizares, M. (2013). Sostenibilidad y turismo: de la documentación internacional a la planificación en España Horizonte 2020. Boletín de la Asociación de Geógrafos Españoles, 61, 67-92. 10.21138/bage.1536

Cardoso, C., Castillo, M. y Hernández, C. (2014). Sosteniendo al turismo o turismo sostenible. Reflexiones teóricas. Estudios y Perspectivas en Turismo, 23, 376-95. ISSN 1851-1732. https://bit.ly/3dMOTql

Carpenter, S. (1991). Inventing sustainables Technologies. The Technology of Discovery and the Discovery of Technology. Blacksburg. https://bit.ly/3exRdzR

Carretero-Díaz, M. A. y Barrientos-Báez, A. (2019). Necesidad de formar a los cuidadores de personas en situación de dependencia: comunicación y respuesta desde la UCM. Revista de Comunicación y Salud, 9(1), 19-38. http://doi.org/10.35669/revistadecomunicacionysalud.2019.9(1).19-38

Castillo, I. y Villagra, N. (2019). La comunicación de la RSE. Análisis del sector hotelero. Revista Latina de Comunicación Social, 74, 1580-1609. 10.4185/RLCS2019-1400

Castrillón, I., Canto, A., Cantorna, A. y Cerradelo, L. (2015). Análisis de los principales modelos explicativos de la competitividad de los destinos turísticos en el marco de la sostenibilidad. CULTUR Revista de Cultura e Turismo, 5(2), 101124. https://bit.ly/2YZG1a]

Comisión de las Comunidades Europeas (2001). El Libro Verde: Fomentar un marco europeo para la responsabilidad social de las empresas. https://bit.ly/2WtQhX7

Comisión Europea (2016). The European Tourism Indicator System (ETIS). https://bit.ly/2WrcvZW

Conde, S. (2017). El turismo sostenible y su importancia en España y a nivel global. Turismo Sostenible En Gipuzkoa: Desafíos y Oportunidades. OPEX: Observatorio de Política Exterior Española, 10-23. ISBN: 978-84-15860-82-2. https://bit.ly/2AnxPXM

Del Rosario, L. (2019). La fibra de las plataneras sirve como bioplástico de uso agrícola. Canarias 7. https://bit./y/2XdUmzn

Díaz, R. y Gutiérrez, D. (2010). La actitud del residente en el destino turístico de Tenerife: evaluación y tendencia. PASOS Revista de Turismo y Patrimonio Cultural, 8(4), 431-444. https://doi.org/10.25145/j.pasos.2010.08.039 
Espinosa, N. y Cazalla, C. (2017). Marco regulatorio del turismo sostenible. Turismo Sostenible En Gipuzkoa: Desafíos Y Oportunidades. OPEX: Observatorio de Política Exterior Española, 24-52. ISBN 978-84-15860-82-2. https://bit.ly/2AnxPXM

Fernández, J. (2015). El Turismo Sostenible en España: Análisis de los Planes Estratégicos de Sostenibilidad en el ámbito local. [Tesis doctoral, Universidade da Coruña]. https://core.ac.uk/download/pdf/61914699.pdf

Fernández, J. (2020). El turismo sostenible en España: análisis de los planes estratégicos de sostenibilidad de Zaragoza y Barcelona. ROTUR, Revista De Ocio Y Turismo, 14(1), 94-106. ISSN-e 2695-6357. https://dialnet.unirioja.es/servlet/articulo?codigo $=7324672$

Gallego Jiménez, G. y Vidal Raméntol, S. (2018). El valor o la virtud en la educación. Vivat Academia. Revista de Comunicación, 145, 23-39. http://doi.org/10.15178/va.2018.145.23-39

Gobierno de España. (2020). El Gobierno Declara La Emergencia Climática. https://bit.ly/2AfIraW

González, M., y Menéndez, A. (2008). Ciencia, tecnología y sostenibilidad. Revista Iberoamericana de Ciencia, Tecnología y Sociedad, 4(11), 47-51. https://www.redalyc.org/articulo.oa?id=92441104

Ivars, J. (2001). Planificación y gestión del desarrollo turístico sostenible: Propuestas para la creación de un sistema de indicadores. Proyecto METASIG, 1, 1-63. ISSN: 1578-679-X. https://bit.ly/2WRILV3

Las siete propuestas de EQUO para que el turismo en España sea sostenible. (2016). El Boletín. https://bit.ly/3cdtFyR

Lorite García, N., Grau Rebollo, J. y De Sousa Lacerda, J. (2018). Representación de la diversidad sociocultural en la publicidad audiovisual: materiales para un tratamiento inclusivo. Revista Latina de Comunicación Social, 73, 425-446. https://doi.org/10.4185/RLCS-2018-1263

Macedo, B. (2005). El concepto de sostenibilidad. Oficina Regional de Educación para América Latina y el Caribe. UNESCO Santiago. 57-59. https://bit.ly/3fGCqEx

Marquardt, B. (2003). Anclando el principio de sostenibilidad, 201-235. https://bit.ly/3eth3V]

Marquardt, B. (2006). Historia de la sostenibilidad. Un concepto medioambiental en la historia de Europa central. Historia Crítica, 32, 172-197. ISSN: 0121-1617. https://doi.org/10.7440/histcrit32.2006.07

Martín, M. y Sánchez, S. (s.f.) Destinos Turísticos Inteligentes: conceptualización, desarrollo e implementación en España. IDITUR-OSTELEA. https://bit.ly/2LpGk6T 
Menzel, H. (2001). El concepto de desarrollo sostenible: el desafío de la legislación y Aplicación de la ley. Revista de política legal, 5, 221-229. Beck.

Ministerio de Economía y competitividad. (2017). Guía del participante en Horizonte 2020. Es Horizonte 2020. https://bit.ly/3bpaPnT

Ministerio de Economía y Hacienda, y Ministerio de Medio Ambiente. (1999). Un turismo sostenible. https://bit.ly/2M7kDJ5

Murcia, C., Ramírez, J., Valderrama, O. y Morales, A. (2017). Turismo sostenible: una conceptualización de su viabilidad para el municipio de Ibagué, desde un estado del arte pertinente. PASOS Revista de Turismo y Patrimonio Cultural, 15, 21-34. https://bit.ly/2XINIjm

Naciones Unidas. (1987). Nuestro futuro común: Informe Brundtland. https://bit.ly/2TfwCsf

Naciones Unidas. (2015). Memoria del Secretario General sobre la labor de la Organización. Asamblea General. https://undocs.org/es/A/70/1

Naredo, J. (1990). La economía y su Medio Ambiente. Ekonomiaz, 17, 12- 25. https://bit.ly/35WFE2c

OMT (2016). Año Internacional del Turismo Sostenible para el Desarrollo. https://bit.ly/2YX6LIV

OMT (2019). Las llegadas de turistas internacionales suman 1.400 millones dos años antes de las previsiones. https://bit.ly/2AjP2Ba

ONU (2000). Declaración del milenio. A/RES/55/2. https://undocs.org/A/RES/55/2

ONU (2015). Memoria del Secretario General sobre la labor de la Organización. ISSN 0252-0036. https://undocs.org/es/A/70/1

Organización internacional del trabajo (2016). ¿Qué es un empleo verde?. https://bit.ly/2BdEKmV

Orozco, J. y Núñez, P. (2013). Las teorías del desarrollo en el análisis del turismo sustentable. Intersedes: Revista de las Sedes Regionales, 14(27), 143-67. ISSN 2215-2458. https://www.redalyc.org/articulo.oa?id=66627452008

Ortega, J. (2019). Tasas turísticas en España y en Europa: lo que hay que pagar. Hosteltur. Edición España. https://bit.ly/2yEGioS

Ortiz F. y Camargo, I. (2010). Propuesta de valores para una cultura organizacional en el turismo sostenible. PASOS Revista de Turismo y Patrimonio Cultural. 8(1), 125-38. https://doi.org/10.25145/j.pasos.2010.08.010 
Plumed, M., Gómez, D. y Martín, C. (2018). Planificación turística, promoción y sostenibilidad ambiental: el caso de España. Retos Revista de Ciencias de la $\begin{array}{llll}\text { Administración y Economía, 15(8), } & \text { 7-18. }\end{array}$ https://doi.org/10.17163/ret.n15.2018.01

Quintero, J. (2004). Los impactos económicos, socioculturales y medioambientales del turismo y sus vínculos con el turismo sostenible. Anales del Museo de América, 12, 263-274. https://bit.ly/3dwQs9Y

Ramírez, G. (2015). La gestión del turismo sostenible: El caso español. [Trabajo final de Grado, Universidad de Cádiz]. https://bit.ly/2WxusGt

Ramón, N. (2015). Vertidos de aguas en Santa Cruz: mucho por depurar. Canarias Ahora Semanal, El Diario. https://bit.ly/2Xafdn6

Redclift, M. (1987). Sustainable development: exploring the contradictions. Community Development Journal, 23. https://doi.org/10.1093/cdj/23.2.130

Rodríguez-Fernández, L. y Vázquez-Sande, P. (2019). Retos y perspectivas en la comunicación organizacional. El profesional de la información, 28(5). https://doi.org/10.3145/epi.2019.sep.01

Salvatierra Iriarte, M. L. C. (2018). Ética en la educación superior en el contexto venezolano para la sostenibilidad organizacional. Revista de Ciencias de la Comunicación e Información, 23(2), 55-65. http://doi.org/10.35742/rcci.2018.23(2).55-65

Sánchez-Marín, F. J., Parra-Meroño, M. C. y Peña-Acuña, B. (2019). Experiencias de trabajo cooperativo en la educación superior. Percepciones sobre su contribución al desarrollo de la competencia social. Vivat Academia. Revista de Comunicación, 147, 87-108. http://doi.org/10.15178/va.2019.147.87-108

Shiva, V. (1989). Staying alive: Women, ecology and development. Zed Books. https://doi.org/10.1111/j.1527-2001.1991.tb00218.x

UNWTO. (2018). Contribution from the countries in the Committee on Tourism Statistics and Tourism Satellite Account. Seventh Meeting of the Inter-Agency and Expert Group On the Sustainable Development Goal Indicators. https://go.aws/2Watmfs

Vogeler, C. y Hernández, E. (2003). El mercado turístico. Estructura, operaciones y procesos de producción. Editorial Centro de Estudios Ramón Areces, S.A. https://bit.ly/2YZgZJ1

VV.AA. (2017). Lloret de Mar trabaja para posicionarse como un destino sostenible. Lloret de mar. https://bit.ly/3brOma0

Wall G. y Mathieson A. (2006). Tourism Change, Impacts and opportunities. Prentice Hall. https://bit.ly/2yT3hwC 
Walls Ramírez, M. (2020). Aportes de la comunicación para la difusión del patrimonio cultural. Revista de Ciencias de la Comunicación e Información, 25(1), 49-55. http://doi.org/10.35742/rcci.2020.25(1).49-55

Zarta, P. (2018). La sustentabilidad o sostenibilidad: un concepto poderoso para la humanidad. Tabula Rasa, 28, 409-423. https://doi.org/10.25058/20112742.n28.18

Zoreda, J. y Perelli, O. (2014). Los 20 años que han revolucionado el sector turístico español. Economía Hosteltur. https://bit.ly/2WuCgZa

\section{AUTORES:}

\section{Almudena Barrientos Báez}

Dra. en Educación y profesora en la Escuela Universitaria de Turismo Iriarte, adscrita a la ULL (Tenerife-España) y en la Univ. Europea. Máster en Dirección de Protocolo, Producción, Organización y Diseño de Eventos - área de Comunicación - (UCJC) y Máster en Dirección de Alojamientos Turísticos (Univ. Girona). Grado en Turismo (EUTI-ULL) y Magisterio (Univ. Valencia).

Forma parte del proyecto DEBATV, Debates Electorales Televisados en España: Modelos, Proceso, Diagnostico y Propuesta (CSO2017-83159-R), proyecto de I+D+I, y del proyecto Nuevos valores, gobernanza, financiación y servicios audiovisuales públicos para la sociedad de Internet: contrastes europeos y españoles (RTI2018096065-B-I00) de investigación del Programa Estatal de I+D+I orientado a los Retos de la Sociedad del Ministerio de Ciencia, Innovación y Universidades (MCIU), Agencia Estatal de Investigación (AEI) y del Fondo Europeo de Desarrollo Regional (FEDER). almudenabarrientos@iriarteuniversidad.es

Orcid ID: https://orcid.org/0000-0001-9913-3353

\section{David Caldevilla-Domínguez}

Licenciado y Doctor en Ciencias de la Información (Comunicación Audiovisual por la U. Complutense). Diplomado en Magisterio (U. de Zaragoza). Profesor titular en la Facultad de Ciencias de la Información de la UCM. Docente en: U. Complutense, U. Europea de Madrid, IED, ESERP e IPAM (Oporto -Portugal-). Índice h $12.6^{\circ}$ autor español en trabajos publicados, $13^{\circ}$ en artículos citados y $20^{\circ}$ en citas recibidas de 747 autores totales.

Ponente y conferenciante en diversos cursos y profesor en varios títulos propios (Telemadrid, Walter \& Thompson, McCann...). Secretario General de la SEECI (Sociedad Española de Estudios de Comunicación Iberoamericana) y del "Fórum Internacional de la Comunicación y Relaciones Públicas" (Fórum XXI).

Investigador Principal (IP) del Grupo Complutense de Investigación 'Concilium'. Autor de más de 70 artículos científicos y de 6 libros: Asturias y La Rioja, una historia común, El sello de Spielberg, Cultura y RRPP, Manual de RRPP, Las RRPP y su fundamentación y La cara interna de la comunicación empresarial. Director de 6 tesis doctorales (2 con mención europea). 
davidcaldevilla@ccinf.ucm.es

Orcid ID: https://orcid.org/0000-0002-9850-1350

\section{Andrea Cáceres Vizcaíno}

Graduada en Turismo en la Universidad de La Laguna.

Curso de especialización en Protocolo y Relaciones Públicas.

Curso de Revenue Management.

Curso de AMADEUS.

Curso de Opera.

caceresvizcainoandrea@gmail.com

\section{Ema Gloria Sueia Val}

Graduada en Turismo en la Universidad de La Laguna.

Curso de especialización en Protocolo y Relaciones Públicas.

Curso de Revenue Management.

Curso de AMADEUS.

Curso de Opera.

gloriasueiaval@gmail.com 